\title{
El Istmo: región, espacio y poder
}

\author{
Elisabeth Cunin y Esteban Krotz
}

Emilia Velázquez, Eric LÉonard, Odile

Hoffmann y Marie-France Prévôt-

SCHAPIRA (COORDS.), 2009

El Istmo mexicano: una región

inasequible. Estado, poderes locales

$y$ dinámicas espaciales (siglos XVI-XXI)

Centro de Investigaciones y Estudios Superiores

en Antropología Social, Institut de la Recherche pour

le Développement, México, 717 pp.

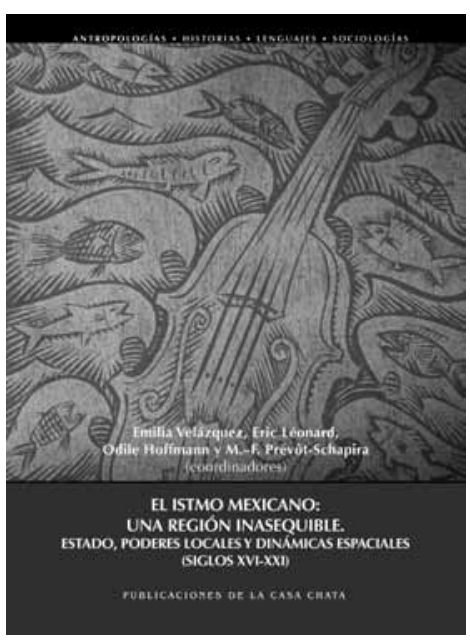
UNA OBRA CONTRES EJES
TEMÁTICOS

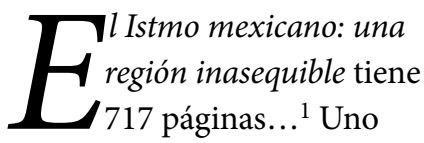

podría enfatizar la abundancia,

${ }^{1}$ Esta reseña resulta de la integración de las partes centrales de las intervenciones que tuvieron los autores en la presentación de la obra el 28 de septiembre de 2010, en el Centro de Investigaciones y Estudios Superiores en Antropología Social-Peninsular, en la ciudad de Mérida.

\section{El Istmo: Region, Space and Power}

Elisabeth Cunin: Instituto de Investigación para el Desarrollo, París, Francia Elisabeth.Cunin@ird.fr

Esteban Krotz: Unidad de Ciencias Sociales, Universidad Autónoma de Yucatán, Mérida, Yucatán, México kroqui@prodigy.net.mx

Desacatos, núm. 39, mayo-agosto 2012, pp. 201-206 la multiplicidad, la minucia de los estudios que contiene. Todo eso es cierto. Pero una de las características más llamativas del libro es que haya logrado construir un análisis unitario y coherente a partir de una diversidad de enfoques, temas, y objetos de estudio en varios años de investigación multidisciplinaria llevada a cabo mediante la cooperación de varias instituciones y grupos de investigación. El libro se compone de cuatro partes, que corresponden a igual número de periodos históricos, en las cuales se estudian los regímenes de control de la población y del territorio, las transformaciones y reconfiguraciones de los actores, de las instituciones, de 
los espacios y de sus

interacciones. Si bien cada parte tiene su especificidad, las cuatro forman un conjunto, dialogan entre sí, buscan contestar las mismas preguntas. Son tres los hilos que vinculan los 13 capítulos del libro: el anclaje de los procesos sociales y de su análisis en el espacio, los juegos de escala región/nación/ globalización y las relaciones de poder entre actores e instituciones.

Durante la presentación del libro en Mérida, una de las coordinadoras del libro y autora de uno de los capítulos enfatizó la importancia de la lógica espacializada de las investigaciones reunidas en esta comentado esta dimensión espacial, sin embargo tan evidente que por ello no se había mencionado. Éste puede considerarse uno de los logros del libro, porque cuando el anclaje espacial de las lógicas sociales se vuelve tan imprescindible para la comprensión del análisis, el lector olvida que es el resultado de una postura metodológica rigurosa, lo mismo que de un meticuloso trabajo de campo y de archivo. Más allá de la riqueza del bagaje conceptual del libro, lo que cada texto hace ver son prácticas situadas, espacios vividos, actores concretos en sus ambientes específicos. El Istmo, antes de ser - o no- una región, aparece así a través de "detalles" prácticos

ordinarios. Estas observaciones, explicitadas y discutidas durante la citada presentación del volumen, son relevantes también en vista de ciertas corrientes en ciencias sociales, muchas veces llamadas "posmodernas", para las que la fascinación por un cierto tipo de "globalización" conduce no solamente a una supuesta “desterritorialización” de los fenómenos culturales, sino también a una especie de evaporización de las relaciones sociales y de los vínculos de la sociedad con la naturaleza.

El segundo tema que une los textos es el de la interdependencia entre región, nación y sociedad global. ¿Se define una región por su dimensión geográfica? ¿Se define en términos de su interacción con el "exterior” y sus fronteras? ¿Es una región una unidad cultural o demográfica? ¿Tiene un desarrollo económico común? ¿Se configura a través de un sistema de comunicación? Estas preguntas son abordadas con enfoques distintos y en varias épocas en los estudios contenidos en el libro. De este modo, la región no sólo se construye a partir de sus características propias -incluso de sus divisiones internas, en particular, del Istmo oaxaqueño y el Istmo veracruzano-, sino también a partir de su relación con el resto de la nación y de su inserción en un universo más amplio marcado desde el inicio de la colonización por lógicas globales - las políticas de la administración española, diversos intereses económicos, lo derivado de la comunicación transístmica-. Sería interesante ubicar esta discusión más adelante de manera explícita en la larga tradición de "estudios regionales" de las ciencias sociales mexicanas (véase, por ejemplo, Palerm, 1972; Viqueira, 2001, y Boehm de Lameiras, 1997). Al mismo tiempo, el tema de una estructura social de la región como tal queda abierto, ya que en los diferentes estudios se identifican ante todo microestructuras en la región.

Un tercer tema omnipresente en el libro es la importancia otorgada por los autores a las relaciones de dominación y de control, lo que incluye el análisis de los espacios de autonomía de los actores y de los procesos de negociación en los que han estado envueltos. De hecho, la región no se desarrolla como resultado de un proyecto concebido de antemano, sino como el resultado - a veces inesperadode las interacciones entre distintos actores, en las que cada uno busca sus propios intereses. De ahí la multiplicidad de conflictos, tensiones, negociaciones, que son motores de los cambios sociales y espaciales. No se elabora una visión lineal de los procesos sociales, al contrario, se presentan y analizan casos de confrontación entre centro y periferia, entre "abajo" y "arriba", que hacen énfasis en las "capacidades de las 
sociedades locales para subvertir los proyectos estatales" (p. 396). En un vaivén permanente entre las reflexiones teóricas y los datos empíricos, el libro propone una serie de conceptos que aclaran las múltiples articulaciones entre dominación y resistencia, las lógicas de "coproducción" de las políticas oficiales por parte de los actores locales: el "Estado local", la "institución local del Estado", la "acción regionalizante del Estado", la "ciudadanía rural", el "anclaje local del Estado" o la "institucionalización local y regional del Estado". Aunque a algunos lectores -en particular a no especialistas en ciencias sociales- estas discusiones pueden parecerles un tanto bizantinas, son ciertamente importantes, ya que el trabajo conceptual es crucial para el análisis de los fenómenos observados. ${ }^{2}$ Además, dicho trabajo conceptual se publica justamente cuando se empieza a hablar, dentro y fuera del país, del Estado mexicano como un Estado "fallido" que parece estar perdiendo progresivamente, y a pesar de la utilización de las fuerzas armadas en el interior de su territorio, la posibilidad de garantizar los derechos fundamentales de los ciudadanos que lo habitan. ${ }^{3}$

\footnotetext{
${ }^{2}$ Para un reciente estudio conceptual que dedica todo un libro a esclarecer un solo concepto, el de cultura política, puede verse Roberto Varela (2005).

${ }^{3}$ Como han destacado la "Declaración del Primer Congreso Nacional de Antropología
}

\section{LOS CONTENIDOS DE LAS CUATRO SECCIONES DE LA OBRA}

Después de destacar los tres ejes que unen el capitulado, conviene señalar las cuatro partes en que se divide el libro. La primera, titulada "El Istmo colonial: porosidades socioterritoriales y espacios de autonomía", agrupa textos de Laura Machuca, Álvaro Alcántara López y Odile Hoffmann. Analiza la organización territorial del Istmo durante la Colonia, haciendo énfasis particular en las rutas de comunicación, en las dinámicas económicas, en la articulación del Istmo con el resto de la colonia y con la metrópoli y en las relaciones de poder. Proporciona un panorama general de dominación e imposición de un orden colonial, en el cual existen, sin embargo, márgenes de libertad y formas de negociación, que se expresan a menudo a través de los múltiples conflictos que caracterizan la época.

La segunda parte, "El Istmo decimonónico: grupos y redes

Social y Etnología" (Ichan Tecolotl, año 21, núm. 242, p. 16, en línea: <http://ciesas.files.wordpress.com $/ 2010 / 10 /$ ichanoctubreweb.pdf $>$ ) y el pronunciamiento de la Asamblea Ordinaria Anual del Consejo Mexicano de Ciencias Sociales ("Más educación y menos violencia", en línea: $<$ http:// www.comecso.com/home/>), no se trata únicamente de un tema para el análisis político y sociocientífico, sino también de un contexto cada vez más limitante para la investigación social en el país. sociales ante el proyecto de integración nacional-liberal" - con trabajos de Marcela Coronado Malagón, Huemac Escalona Lüttig, Emilia Velázquez y Alfredo Delgado Calderón-, estudia los proyectos estatales de modernización e integración del Istmo, de inserción en una economía internacional, de desarrollo de vías de comunicación, en específico el Ferrocarril de Tehuantepec, de cambios en la propiedad de la tierra y la definición de estas modalidades - tierras nacionales, haciendas, terrenos comunales de indígenas, terrenos baldíos-, de colonización y reconfiguración de los grupos sociales. La tercera parte, "El Istmo posrevolucionario (1): agrarismo oficial y fronteras internas en la construcción de los espacios rurales" - con exposiciones en coautoría a cargo de Eric Léonard, Emilia Velázquez y Aurélia Michel—, se centra en la primera mitad del siglo xx y en la cuestión del reparto agrario. El reto para el Estado posrevolucionario fue construir un nuevo modelo de regulación político-territorial que permitiera desmantelar dos formas de organización que dominaban el campo rural desde la Colonia: la comunidad indígena y la hacienda. Finalmente, la cuarta parte, "El Istmo posrevolucionario (2): culturas y espacios petroleros allende los avatares del régimen 
corporativista" - con capítulos de Marie-France PrévôtSchapira, Manuel Uribe y Saúl Horacio Moreno Andrade-, se ubica a mitades-finales del siglo $\mathrm{xx}$ y estudia las transformaciones ligadas a la industria petrolera y a la urbanización: arreglos clientelistas, arraigo de los líderes a nivel local, régimen corporativista y sindicalista, resistencia/inserción indígena.

\section{CUATRO TEMAS PARA LA DISCUSIÓN}

En una lectura más personal y orientada por los intereses de investigación de quienes escriben esta reseña, llaman la atención de cuatro aspectos. Primero hay que subrayar el carácter colectivo del libro, la articulación entre problemática común y especificidad de cada texto. Si bien el punto de partida es el concepto de "región", también desde el principio se cuestiona esta noción. Incluso uno de los primeros planteamientos es que el estudio de la "región" no debe quedarse sólo entre las manos de los geógrafos humanos, sino que su análisis necesita un enfoque pluridisciplinario. Es así como una interrogante inicialmente geográfica lleva a los autores del libro a otras de carácter histórico, social, económico, político y cultural. El libro empieza con una introducción general que permite tener claridad sobre los objetivos generales, los enfoques teóricos, la definición de los conceptos fundamentales y las preguntas que orientan el estudio (p. 23). Cada parte comienza con una pequeña introducción que le da sentido al conjunto de textos, que orienta y ayuda a la lectura, que retoma los puntos centrales. Más allá de estos notables esfuerzos formales, se observa una dinámica de trabajo colectivo, unas preguntas e inquietudes comunes, al igual que el conocimiento de los trabajos de los otros autores. La obra es el resultado de numerosos intercambios, a largo plazo, entre distintos investigadores y los textos se articulan de hecho como las piezas de un rompecabezas. En este sentido, hay que resaltar la calidad y multiplicidad de los mapas, ${ }^{4}$ que son en general fruto de una colaboración entre varios investigadores, en particular geógrafos e historiadores. Permiten ubicar, materializar y ver de otra manera los planteamientos desarrollados en los textos, así como confrontar los mapas a lo largo de tres siglos. Al final, el lector tiene una imagen coherente y con sentido del Istmo, no porque se reduzca una realidad compleja a unos cuantos elementos, sino porque: 1) los capítulos tienen un marco

\footnotetext{
${ }^{4}$ Lamentablemente, la manera de imprimirlos, en especial su tamaño, les resta importancia a veces.
}

de referencia común, unas preguntas compartidas; y 2) son articulados entre sí, dialogan, se contestan, en un proceso acumulativo de producción colectiva de conocimiento, que integra a mexicanos y extranjeros, investigadores confirmados y jóvenes, historiadores, geógrafos, antropólogos y sociólogos.

Otro elemento que parece pertinente es la reflexión sobre las dinámicas de las relaciones interétnicas y el mestizaje. No es un objeto de estudio explícito del libro, pero varios artículos abordan el tema de manera secundaria. Se introduce una interesante innovación heurística: no se estudia lo indígena o lo afro en sí, sino su relación con otras dinámicas socioespaciales - las consecuencias del ferrocarril sobre la organización de las comunidades indígenas, la integración de la mano de obra zapoteca a la industria petrolera, la transición entre distintos modelos de administración de la población y del espacio, etc.-. Lo étnico se entiende sólo en relación con los contextos sociales en los cuales tiene significación, se moviliza, se invisibiliza y se visibiliza, no se define a partir de unos criterios propios "internos" de tal o cual "grupo", sino en la actualización de ciertos criterios definidos como propios en determinados contextos sociales. La "negociación", en la que se hace 
tanto énfasis a lo largo del libro, no es solamente una negociación entre actores o grupos, es también una negociación del estatus, de la identidad, de la definición misma de estos actores. En este sentido, los trabajos aportan elementos de análisis acerca de los procesos de mestizaje o "mestización” y, así, de la construcción de una nación/ región mestiza. No se interesan por la ideología del mestizaje, objeto de numerosas investigaciones, tampoco por los individuos o grupos que entran - o no- en la dinámica del mestizaje: abren el camino al estudio de las políticas y prácticas del mestizaje. De hecho, se habla de una identidad mestiza que "marginaliza las opciones indígenas" y "borra"

otras identificaciones posibles, entre ellas la de los negros y mulatos en la parte veracruzana del Istmo (p. 165 y ss.), de indígenas que se vuelven ciudadanos (p. 455 y ss.), del proceso de "zapotequización" de los migrantes en la ciudad de Minatitlán (p. 635 y ss.), etc. Todos estos procesos no son contradictorios, ni mutuamente excluyentes, sino que contribuyen a dibujar el mestizaje en la dinámica misma de las reconfiguraciones sociales.

En tercer lugar, El Istmo mexicano... abre una pista hacia posibles comparaciones con otras "regiones", en particular con otra región "inasequible": la Península de Yucatán. Como si no fueran suficientes estas 717 páginas, esta reseña es una invitación a acompañar la lectura del libro sobre el Istmo con la del volumen coordinado por Gabriel Aarón Macías Zapata (2004) sobre - parte de- la Península de Yucatán: El vacío imaginario: geopolítica de la ocupación territorial en el Caribe oriental mexicano. Esta lectura paralela podría tener dos dimensiones. Por un lado, nos permite hacer comparaciones entre las dos regiones, entre los procesos de definición y conformación de las dos entidades. Por ejemplo, es muy llamativo que en ambos libros se haga referencia a las nociones de vacío, existente o creado, y también de regiones "inasequibles", de difícil acceso; o a las nociones de frontera, de tierra de confines, de zona de colonización. Sería interesante confrontar las lógicas sociales a las que hacen referencia estos términos. ¿El vacío, la frontera, el carácter inhóspito tienen el mismo sentido en el Istmo y en la Península? ¿Remiten a dinámicas sociales, a actores, a interacciones similares? Sería pertinente asimismo comparar las formas de imposición del Estado "nacional" y de resistencias locales, de integración de los indígenas, las etapas de desarrollo económico. Por otro lado, dicha lectura abre la posibilidad de estudiar las relaciones entre estas dos "regiones". El libro nos presenta análisis muy detallados sobre la construcción de la región, sobre la relación entre región y nación, incluso sobre su inserción en dinámicas transnacionales o globales, también sobre las dinámicas sociales internas a la región misma. A esto se agregaría otra, es decir, la de los intercambios entre distintas regiones. Por ejemplo: ¿cuáles son las consecuencias, en ambas "regiones", de las migraciones de Veracruz a Quintana Roo, desde principios del siglo pasado - cortadores de madera, chicleros- a la década de 1970 - colonización dirigida para poblar el naciente Estado—? ¿Las dinámicas regionales tienen alguna influencia sobre la definición de la nación? ¿Contribuyen así a "hacer la nación", y no sólo a integrarse en ella?

Finalmente, hay que destacar que este libro es uno de los resultados de un proyecto de investigación de diez años de duración (1996-2006), en el cual colaboraron investigadores de la Unidad Golfo ciesas y del Institut de la Recherche pour le Développement (IRD). En 2001, este equipo multidisciplinario y biinstitucional fue invitado a participar en el proyecto de investigación "El Istmo de Tehuantepec en el contexto actual del desarrollo", de cinco años de duración, apoyado por el Consejo Nacional de Ciencia y Tecnología (Conacyt) y dirigido por el doctor Hipólito Rodríguez de la 
mencionada unidad del CIESAs. ${ }^{5}$

A partir de 2004, la doctora

Emilia Velázquez, una de las editoras del volumen, organizó un seminario de análisis de material — de dos años de duración-, a varias de cuyas sesiones asistieron investigadora(es) de la contraparte francesa. El resultado es, como ya se señaló al inicio de esta reseña y de este apartado, una obra voluminosa pero bien integrada por capítulos cuidadosamente elaborados. Lo anterior significa que el libro $-y$ la investigación que lo produjo- se realizó en cierto sentido a contracorriente de los criterios "de evaluación" que actualmente, $y$ desde hace do impuestos por determinadas instancias federales de ciencia y tecnología y sus representantes en las universidades y centros de investigación: el ritmo de la investigación siguió la dinámica científica e intelectual y no el ritmo anual tan apreciado por los aparatos administrativos. El

\footnotetext{
${ }^{5}$ En el portal electrónico (<http://www.ciesas-golfo.edu.mx/istmo/index.html $>$ ) hay más información sobre el Proyecto Conacyt propiamente dicho. Llama la atención el gran número de tesis de licenciatura y maestría (varias de las cuales están accesibles en línea, más otros textos de reuniones, ponencias, documentos de trabajo). Lamentablemente, el libro carece de un apartado específico dedicado a la descripción y autoevaluación de la organización del trabajo en el proceso de investigación y el análisis de material.
}

"trabajo colectivo", celebrado por funcionarios de todos los niveles de instituciones académicas desde hace algún tiempo como algo

intrínsecamente valioso, no se simuló ni se llevó a cabo de forma abstracta, sino que ocurrió de diferentes maneras en función de las diferentes fases de la investigación $-y$ fue posible en la fase final por cuantiosos recursos "solamente" para discutir ideas y textos-. El trabajo "en red" no se hizo con la finalidad principal de legitimarse ante instancias evaluadoras otorgadoras de "puntos", sino para confrontar hipótesis, modelos y borradores cuando fuera necesario, y en vez del "artículo" —o sea, el texto relativamente corto, de autoría preferentemente colectiva y publicado fuera del país y en cualquier idioma menos el castellano, el cual para no pocas instancias de la administración universitaria y científica nacional parece ser el único resultado respetable de una investigación científica propiamente dichase produjo un voluminoso libro compuesto por capítulos integrados y textos que los vinculan entre sí. ${ }^{6}$ Desde ahora,

\footnotetext{
${ }^{6}$ Podría decirse también que se trata de un acto de resistencia ante la amenaza de ser engullida por la burocracia digitalizada, la hegemonía del concepto de ciencia de las llamadas ciencias naturales o exactas y el modelo gerencial-cuantitativista de la universidad (Krotz, 2009: 84 y ss.).
}

el libro tiene asegurado su lugar en los esfuerzos de especialistas para entender el Istmo mexicano $y$, es de desearse, también de quienes viven en la región y de quienes toman decisiones sobre ella y sus moradores.

\section{Bibliografía}

Boehm de Lameiras, Brigitte, 1997, "El enfoque regional y los estudios regionales en México: geografía, historia y antropología", en Relaciones, vol. XVIII, núm. 72, pp. 15-46.

Krotz, Esteban, 2009, "Hacia la recuperación del lugar de las ciencias sociales en la sociedad de conocimiento en México", en Revista Mexicana de Ciencias Sociales, año 71, núm. 5, pp. 75-104.

Macías Zapata, Gabriel Aarón (coord.), 2004, El vacío imaginario: geopolitica de la ocupación territorial en el Caribe oriental mexicano, Centro de Investigaciones y Estudios Superiores en Antropología Social, Congreso del Estado de Quintana Roo, México.

Palerm, Ángel, 1972, "Ensayo crítico al desarrollo regional en México", en David Barkin (comp.), Los beneficiarios del desarrollo regional, Secretaría de Educación Pública, sEP-Setentas, México, pp. 33-42.

Varela, Roberto, 2005, Cultura y poder, Universidad Autónoma Metropolitana-Iztapalapa, Anthropos, México, Barcelona.

Viqueira, Carmen, 2001, El enfoque regional en antropología, Universidad Iberoamericana, México. 